\title{
Oferta y demanda de estudios de equivalencia terapéutica (in vitro e in vivo) de medicamentos en Costa Rica
}

Offer and demand of therapeutic equivalence studies (in vitro and in vivo) for medicines in Costa Rica

\section{Zacarías Pereira-Vega ${ }^{1}$}

Fecha de recepción: 27 de mayo del 2015

Fecha de aprobación: 18 de setiembre del 2015

Pereira-Vega, Z. Oferta y demanda de estudios de equivalencia terapéutica (in vitro e in vivo) de medicamentos en Costa Rica. Tecnología en Marcha. Vol. 29, № 1, Enero-Marzo. Pág 18-27. 


\title{
Palabras clave
}

Laboratorio fabricante de medicamentos; estudios de equivalencia terapéutica de medicamentos; intercambiabilidad; medicamento genérico; medicamento innovador; bioexención; medicamento de riesgo sanitario.

\section{Resumen}

El continuo incremento del gasto en medicamentos a nivel mundial, íntimamente asociado al crecimiento poblacional y al aumento de la esperanza de vida de las personas, ha llevado en las últimas décadas a los gobiernos a buscar alternativas que contribuyan a reducir el impacto de este rubro en los presupuestos. Una alternativa es el uso de medicamentos genéricos, que son más baratos que los innovadores. Sin embargo, antes de ser registrado, el producto genérico debe demostrar su equivalencia terapéutica mediante ensayos in vitro y/o in vivo contra un medicamento de referencia, con el propósito de garantizar su calidad, seguridad y eficacia y, como consecuencia de ello, su intercambiabilidad.

Mediante una encuesta online realizada en Costa Rica durante los meses de marzo y abril de 2013, se determinó que existía una demanda de 85 medicamentos fabricados en el país, que para ser registrados a nivel nacional requerían de la misma cantidad de estudios de equivalencia terapéutica, de los cuales 47 correspondían a ensayos in vitro y 38 a ensayos in vivo. Esta demanda provenía principalmente de cuatro laboratorios que fabrican medicamentos en Costa Rica, y aumenta si se consideran los medicamentos exportados. En cuanto a la oferta nacional de estos servicios (ensayos in vitro e in vivo) por parte de laboratorios ajenos a la casa fabricante, se sabe que es muy limitada, ya que solo había dos que los ofertaban y su labor se circunscribe, por ahora, a los ensayos in vitro.

\section{Keywords}

Medicine manufacturing laboratory; therapeutic equivalence study for medicines; interchangeability; generic; originator; biowaiver; sanitary risk medicine.

\begin{abstract}
In the last decades the constant increase of medicine expenses worldwide, as well as the population growth and the increase of life expectancy, has led governments to look for alternatives to help reduce the impact of this matter in their budgets. Generic medicines are one option because they are cheaper than originator medicines. Nevertheless, the generic medicine must prove its therapeutic equivalence, before its registration, by undergoing in vitro and/or in vivo essays compared to a reference medicine to guarantee its quality, safety and efficacy; and, thus, its interchangeability.

An on line survey executed in March and April, 2013, helped determine there was a demand for 85 medicines produced in Costa Rica that, to be registered in the country, required the same amount of therapeutic equivalence studies; 47 required in vitro essays and 38 required in vivo essays. This demand came mainly from four laboratories that produce medicines in the country; it increases if exported medicines are included. Regarding the national offer of these services (in vitro and in vivo essays) from laboratories that do not belong to the producer, it is well known that this is very limited, since there were only two laboratories offering the service, and their work is limited, for now, to in vitro essays.
\end{abstract}




\section{Introducción}

El consumo de medicamentos a escala mundial aumenta año con año y va de la mano con el aumento de la población y la esperanza de vida de las personas. Este asunto en alguna medida representa una verdadera preocupación para los sistemas de seguridad social y las autoridades sanitarias, ya que el gasto en medicamentos es significativo y crece aceleradamente (IMS Institute, 2011). Al menos en Costa Rica, el gasto en medicamentos realizado por la Caja Costarricense del Seguro Social (CCSS) -el mayor importador y consumidor de estos bienes en el país- en el año 2011 superó los 101,000 millones de colones (CCSS, 2012), lo que representaba, según el tipo de cambio oficial de entonces, un monto cercano a US\$198 millones. Los medicamentos son el recurso sanitario más empleado y uno de los productos más regulados por la administración pública de cada uno de los países del orbe. Todo esto lleva a una discusión de interés público sobre la elección entre los medicamentos innovadores y los genéricos. Estos últimos también se conocen en el mercado farmacéutico como medicamentos multiorigen o multifuente. Dado que el término medicamento genérico es el más conocido por quienes no son profesionales en Farmacia, este artículo se referirá a ellos de esta manera.

Los medicamentos innovadores son aquellos en los que un laboratorio farmacéutico ha invertido importantes recursos en investigación y desarrollo durante cierto período de tiempo, realizando estudios de síntesis química, estudios preclínicos, clínicos, galénicos y de biodisponibilidad, para efectos de documentar y, consecuentemente, garantizar su calidad, seguridad y eficacia (EFPIA, 2007). Para que un medicamento innovador pueda ser comercializado, debe pasar al menos, en promedio, un período de entre diez y veinte años desde su descubrimiento inicial. Se puede decir entonces que este tipo de medicamento es el primero que se comercializa y, además, cuenta con una patente a nivel mundial que le garantiza al laboratorio farmacéutico innovador su fabricación y comercialización monopolística durante cierto período de tiempo, que suele ser de 10 años, lo que le permite recuperar su inversión inicial. Es importante mencionar que el laboratorio farmacéutico innovador comercializa su producto con un nombre comercial propio, también conocido como nombre de marca.

En la legislación costarricense, la definición de medicamento genérico es similar a la dada por la Organización Mundial de la Salud (OMS), que lo define como "aquellas alternativas farmacéuticas que pueden o no ser equivalentes terapéuticos. Los equivalentes terapéuticos son intercambiables" (OPS, 2011a). Asimismo, se puede agregar que es todo aquel medicamento que se desarrolla a partir de un medicamento innovador y que contiene la misma composición cualitativa y cuantitativa en cuanto al principio o principios activos, en la misma cantidad o concentración, la misma forma farmacéutica, la misma dosis y las mismas características cinéticas, dinámicas y técnicas que las del producto innovador. Además, en algunos países, como España, Brasil y México, la legislación incorpora en esta definición la obligatoriedad de que el medicamento genérico demuestre su bioequivalencia con el medicamento de referencia mediante estudios adecuados de biodisponibilidad (Vacca et al., 2006), también conocidos como estudios de bioequivalencia. El medicamento de referencia que se señala en esta última definición se refiere, por lo general, al medicamento o producto innovador. Es importante destacar que el medicamento genérico empezará su comercialización hasta el vencimiento de la patente del medicamento innovador, no antes.

Una de las principales ventajas de los medicamentos genéricos sobre los innovadores es que son más baratos, tanto para el paciente como para los sistemas sanitarios (Dunne et al., 2013), ya que la inversión económica que hace el laboratorio farmacéutico que lo propone, para efectos de su desarrollo y comercialización, es significativamente menor que la realizada para los medicamentos innovadores. A este nivel, ya no es necesario demostrar la eficacia y la favorable relación beneficio-riesgo del producto, ni descubrir las indicaciones terapéuticas, 
sus efectos secundarios, contraindicaciones, ni la pauta de administración (dosificación) más apropiada, ya que son aspectos que han sido demostrados previamente por el fabricante del producto innovador. Asimismo, desde el punto de vista de la información y promoción comercial del producto, el esfuerzo que se debe realizar es menor, ya que su principio activo es de sobra conocido. Otra ventaja que presentan los productos genéricos es que su nombre coincide con el del principio activo del medicamento innovador, lo que facilita su identificación por parte de los profesionales sanitarios. Además, como última ventaja se puede agregar que la aparición de los genéricos en el mercado afecta de manera positiva la oferta, y como consecuencia, se genera una reducción en los precios del medicamento innovador (Abad et al., 2008) y de los demás que contengan el mismo principio activo.

Por otro lado, la principal desventaja de los medicamentos genéricos con respecto a los innovadores es el riesgo de la mala adherencia al tratamiento, ya que con frecuencia el genérico presenta una forma y un color diferentes de los del medicamento innovador, lo que en alguna medida confunde al paciente, especialmente en el caso de ancianos y/o polimedicados que reciben tratamientos para enfermedades crónicas. Para solucionar este problema, algunos especialistas coinciden en que sería conveniente que el medicamento genérico conserve la forma y el color que posee el innovador, lo que se conoce como bioapariencia del producto. Sin embargo, la mayoría de las veces esto no es posible, dado que muchas formas y colores de los medicamentos ya han sido patentadas.

Dada esta situación entre medicamentos genéricos e innovadores, desde hace algunos años en la gran mayoría de los países se ha abierto la discusión en torno a la conveniencia de la intercambiabilidad de los medicamentos, dado el menor costo que presentan los genéricos y, en consecuencia, el impacto positivo que producen en los presupuestos de salud pública de las naciones. Por otro lado, no se pueden pasar por alto los errores de falla terapéutica de medicamentos, provocados por medicamentos mal formulados o por el uso inadecuado de principios activos y excipientes. Es por tal razón que los genéricos deben ser medicamentos de calidad, seguridad y eficacia demostradas.

Para que un medicamento genérico sea intercambiable, tiene que demostrar su equivalencia terapéutica con un producto de referencia. Dependiendo de la ubicación del principio activo en el Sistema de Clasificación Biofarmacéutica (SCB), que está íntimamente relacionada con sus propiedades fisicoquímicas (solubilidad y permeabilidad a través de las membranas del tracto gastrointestinal), la equivalencia terapéutica se puede demostrar mediante estudios in vitro y/o in vivo (Moreno, 2011). Los estudios in vitro también se conocen como perfiles de disolución comparativos, mientras que los estudios in vivo se denominan estudios de bioequivalencia. Los estudios in vitro corresponden a curvas de disolución del principio activo en función del tiempo, que se realizan bajo condiciones de temperatura controlada $\left(37^{\circ} \mathrm{C}\right)$ y $\mathrm{pH}$ controlado $(1,2,4,5$ y 6,8), así como otros requerimientos (FDA, 2010). Es importante aclarar que, por lo general, los estudios in vitro son complementarios, que por sí solos no garantizan que un medicamento sea equivalente terapéutico con un producto de referencia. Por otro lado, los estudios de bioequivalencia (estudios in vivo) se realizan mediante la implementación de ensayos clínicos comparativos en voluntarios sanos, que corresponden a estudios farmacocinéticos y/o farmacodinámicos (Niazi, 2007). Bajo esta perspectiva, cuando un medicamento genérico demuestra su equivalencia terapéutica con respecto al medicamento de referencia, el primero no exhibe diferencias significativas en la cantidad y velocidad de absorción del principio activo con respecto al segundo. Esto es lo que garantiza, a final de cuentas, la intercambiabilidad entre ambos productos.

La OMS recomienda a todos sus países miembros tender hacia la demostración de la equivalencia terapéutica de los medicamentos y la consecuente declaración de intercambiabilidad de los productos genéricos (OPS, 2011b). De la misma manera, la OMS también recomienda 
establecer los criterios básicos para los estudios de equivalencia terapéutica de medicamentos (in vitro e in vivo), que son necesarios para asegurar la intercambiabilidad de los genéricos. Por otro lado, también se tienen que tener presente los criterios para las exenciones de los estudios in vivo, conocidos como bioexenciones o bioexcepciones. Estas bioexenciones se establecen considerando el SCB (OPS, 2011b), el cual divide o clasifica a los medicamentos en cuatro clases, según su grado de solubilidad y permeabilidad. De acuerdo con este sistema, que fue introducido en 1995, los medicamentos de la clase I presentan alta solubilidad y alta permeabilidad, los de la clase II son de baja solubilidad y alta permeabilidad, los de la clase III son de alta solubilidad y baja permeabilidad y los de la clase IV presentan baja solubilidad y baja permeabilidad (Saavedra et al., 2011; FIP, 2012). Las bioexenciones se dan principalmente con los medicamentos de la clase I (alta solubilidad y alta permeabilidad) que se liberen rápidamente (más del 85\% en 30 minutos), aunque también se puede extender los criterios de bioexención a algunos de los medicamentos de la clase II (baja solubilidad y alta permeabilidad) y de la clase III (alta solubilidad y baja permeabilidad) que cumplan ciertas características en cuanto a la rapidez de disolución y la similitud de los perfiles de disolución comparativos (WHO, 2006). En los casos en que se cumplan los criterios de bioexención, el producto probará su equivalencia mediante estudios in vitro, siempre que no pesen sobre él las consideraciones de medicamento de riesgo sanitario alto (alta probabilidad de provocar eventos adversos en el paciente o incluso ocasionar la muerte) o que presente un margen terapéutico estrecho (dosis terapéutica muy cercana a la dosis tóxica, lo que puede provocar daño en el paciente e incluso la muerte).

Dado que los estudios de equivalencia terapéutica de medicamentos no son baratos, en especial los de bioequivalencia (in vivo), y, por lo tanto, podrían influir en su costo o precio final, en Costa Rica estos estudios se exigen solo a aquellos que presentan un riesgo sanitario alto o intermedio o bien un rango o margen terapéutico estrecho.

Es importante destacar que puede ser que dos presentaciones farmacéuticas que contengan el mismo principio activo, en la misma dosis y en la misma formulación, no sean bioequivalentes, y la causa puede deberse a diferencias en las materias primas (tanto de los principios activos como de los excipientes), o a diferencias en las distintas etapas de los procesos de formulación, transposición de escala y/o de fabricación a escala industrial. Estos aspectos, actuando solos o en conjunto, pueden alterar de manera significativa la velocidad de disolución y/o la absorción del principio activo a través de las membranas del tracto gastrointestinal.

Un principio básico fundamental que rige los estudios de bioequivalencia de medicamentos es el siguiente: "a igual biodisponibilidad (velocidad y cantidad de principio activo absorbido y que llega al tejido o área donde se produce su efecto), se obtienen efectos farmacológicos iguales" (Abad et al., 2008). De la misma manera, se entiende que existe bioequivalencia entre dos medicamentos (genérico y referencia) cuando presentan una biodisponibilidad similar en condiciones experimentales apropiadas. Por otro lado, existe un acuerdo internacional que dice que dos formulaciones (medicamento genérico y referencia) son bioequivalentes cuando la diferencia en lo concerniente a la velocidad de absorción y a la cantidad de principio activo absorbido, entre las respectivas a ambas formulaciones, son inferiores al 20\% (Moreno et al., 2010).

En el presente artículo se discute sobre la realidad costarricense en torno a la oferta y demanda de estudios de equivalencia terapéutica de medicamentos, que son servicios esenciales para garantizar la calidad, seguridad y eficacia de los medicamentos genéricos producidos en el país. 


\section{Metodología}

La información para este estudio se obtuvo a partir de una encuesta que se realizó a representantes de los laboratorios fabricantes radicados en Costa Rica, y que requieren de la realización de estudios de equivalencia terapéutica de medicamentos como un requisito para su registro sanitario. La encuesta se realizó vía online en el período comprendido entre los meses de marzo y abril de 2013, y se usó para estos propósitos el programa de software libre denominado LimeSurvey® versión 2.00.

\section{Resultados y discusión}

\section{Demanda}

La demanda de estudios de equivalencia terapéutica (in vitro e in vivo) de medicamentos en Costa Rica está relacionada con todo aquel laboratorio nacional (o radicado en el país) fabricante de medicamentos que requiere de este tipo de ensayos para efectos del registro sanitario de sus productos ante el Ministerio de Salud.

Con el fin de determinar la demanda real de estudios de equivalencia terapéutica de medicamentos por parte de los fabricantes en el país, se consultaron diez laboratorios mediante la encuesta, de los que solo contestaron cinco. De la información obtenida, cuatro laboratorios indicaron que sí requerían de estudios de equivalencia terapéutica y uno manifestó que no. A continuación, se dan a conocer los resultados de la encuesta, encaminada a determinar, primeramente, la demanda nacional de estos ensayos durante el período en que se realizó este estudio.

En el cuadro 1 se presentan los resultados al preguntar a los laboratorios sobre la cantidad de los medicamentos que ellos fabrican y que a su vez requieren de estudios in vitro e in vivo, según el país o región a la que son destinados o exportados.

Cuadro 1. Cantidad de medicamentos de los laboratorios fabricantes que requieren estudios in vitro e in vivo según la región a la que son exportados o destinados.

\begin{tabular}{|c|c|c|}
\hline Región & $\begin{array}{l}\text { Número total de } \\
\text { productos (in vitro) }\end{array}$ & $\begin{array}{l}\text { Número total de } \\
\text { productos (in vivo) }\end{array}$ \\
\hline Costa Rica & 47 & 38 \\
\hline Resto de América Central & 72 & 65 \\
\hline Suramérica & 80 & 36 \\
\hline El Caribe & 20 & 13 \\
\hline México & 0 & 0 \\
\hline Estados Unidos y Canadá & 0 & 0 \\
\hline Europa & 0 & 0 \\
\hline Otros continentes & 0 & 0 \\
\hline SUBTOTALES & 219 & 152 \\
\hline TOTAL & \multicolumn{2}{|c|}{371} \\
\hline PORCENTAJE & 59,0 & 41,0 \\
\hline
\end{tabular}

Fuente: Datos obtenidos a partir de la encuesta. 
En el cuadro 2 se señalan diez principios activos -que se nombran según su Denominación Común Internacional (DCl)- como ejemplos de medicamentos correspondientes a formas farmacéuticas orales sólidas, que pertenecen a una lista de más de cuarenta principios activos para los que Costa Rica exige estos estudios como requisito para su registro sanitario ante el Ministerio de Salud.

Cuadro 2. Lista de diez medicamentos para los que Costa Rica exige estudios de equivalencia terapéutica para su registro sanitario.

\begin{tabular}{|c|c|c|c|}
\hline Principio activo (DCI) y fuerza & Categoría terapéutica & Forma farmacéutica & Tipo de ensayo \\
\hline $\begin{array}{l}\text { Carbamazepina } \\
200 \text { mg }\end{array}$ & Antiepiléptico & Tabletas & In vivo \\
\hline $\begin{array}{l}\text { Digoxina } \\
0,25 \mathrm{mg}\end{array}$ & Cardiotónico, antiarrítmico & Tabletas & In vivo \\
\hline $\begin{array}{l}\text { Lamivudina } \\
150 \text { mg }\end{array}$ & Antiviral & Tabletas & In vitro \\
\hline $\begin{array}{l}\text { Efavirenz } \\
200 \text { mg }\end{array}$ & Antiviral & Cápsulas & In vivo \\
\hline $\begin{array}{l}\text { Warfarina sódica } \\
1 \mathrm{mg} \text { y } 5 \text { mg }\end{array}$ & Anticoagulante & Tabletas & In vivo \\
\hline $\begin{array}{l}\text { Amiodarona } \\
200 \text { mg }\end{array}$ & Antiarrítmico & Tabletas & In vivo \\
\hline $\begin{array}{l}\text { Carvedilol } \\
6,25 \mathrm{mg}\end{array}$ & Antihipertensivo & Tabletas & In vivo \\
\hline $\begin{array}{l}\text { Fluoxetina } \\
20 \mathrm{mg}\end{array}$ & Antidepresivo & Tabletas & In vivo \\
\hline $\begin{array}{c}\text { Atenolol } \\
50 \text { mg y } 100 \text { mg }\end{array}$ & Antihipertensivo & Tabletas & In vitro \\
\hline $\begin{array}{l}\text { Clozapina } \\
100 \text { mg }\end{array}$ & Antipsicótico & Tabletas & In vivo \\
\hline
\end{tabular}

Fuente: Datos obtenidos a partir de FIP, 2012 y WHO, 2006.

En el cuadro 3 se presentan los resultados de la pregunta sobre la capacidad del laboratorio fabricante -en cuanto a personal técnico e instrumental- para realizar estudios in vitro e in vivo de los medicamentos que producen.

Cuadro 3. Capacidad del laboratorio fabricante para realizar estudios in vitro e in vivo.

\begin{tabular}{|c|c|c|}
\hline Tipo de estudio & Sí & No \\
\hline In vitro & 4 & 0 \\
\hline In vivo & 2 & 2 \\
\hline
\end{tabular}

Fuente: Datos obtenidos a partir de la encuesta. 
En el Cuadro 4 se presentan los resultados de la pregunta sobre quién realiza los estudios in vitro e in vivo de los medicamentos que sus laboratorios fabrican.

Cuadro 4. ¿Quién realiza los estudios in vitro e in vivo?

\begin{tabular}{|c|c|c|c|}
\hline Tipo de estudio & El propio laboratorio & $\begin{array}{r}\text { Se contrata una } \\
\text { empresa }\end{array}$ & $\begin{array}{c}\text { Ambas (el propio laboratorio } \\
\text { y empresa contratada) }\end{array}$ \\
\hline In vitro & 2 & 0 & 2 \\
\hline In vivo & 0 & 3 & 1 \\
\hline
\end{tabular}

Fuente: Datos obtenidos a partir de la encuesta.

De la información contenida en el cuadro 1 se puede afirmar que los cuatro laboratorios fabricantes de medicamentos que contestaron completamente la encuesta requieren de los estudios de equivalencia terapéutica, no solo para efectos de su registro sanitario en Costa Rica sino para aquellos que exportan a otros países, dado que también se les exige este tipo de ensayos. Asimismo, los principales mercados a los que están dirigidos estos medicamentos son América Central, El Caribe y Suramérica. Por otro lado, los laboratorios consultados manifestaron en ese momento que había un universo de 371 productos que requerían de estudios de equivalencia terapéutica, de los cuales 219 corresponden a estudios in vitro y 152 a estudios in vivo. En este aspecto, es importante aclarar que estas cifras pueden ser nominales, ya que uno o varios medicamentos de un mismo fabricante coinciden en su necesidad de estos estudios en varios países. Sin embargo, del mismo cuadro se puede extraer la demanda real de estudios de equivalencia terapéutica de medicamentos en Costa Rica durante el período mencionado, que asciende a un total de 85, de los cuales 47 corresponden a estudios in vitro y los 38 restantes a estudios in vivo.

En el cuadro 3 se evidencia que los cuatro fabricantes dicen tener la capacidad para realizar perfiles de disolución comparativos (estudios in vitro) y la mitad de ellos, o sea dos, afirman lo mismo en cuanto a los estudios de bioequivalencia (estudios in vivo).

Con respecto a la información del cuadro 4, para los estudios in vitro, dos laboratorios manifiestan realizar sus propios estudios, mientras que los otros dos indican hacerlos ellos mismos o contratar una empresa (laboratorio de ensayo) externa. Para los estudios in vivo, tres laboratorios indican que contratan una empresa (laboratorio de ensayo) y el otro manifiesta esta opción o poder realizarlos ellos mismos. Se entiende que esto se refiere a que se hace fuera del país, dado que la legislación no permitía entonces la realización de estos estudios en Costa Rica. Dada esta situación con los estudios in vivo, los fabricantes de medicamentos nacionales han recurrido a otros países para subsanar esta deficiencia en la legislación y que afecta o afectaba hasta hace muy poco tiempo la oferta de los laboratorios de ensayo costarricenses que brindan estos servicios. Algunos de estos países son México, Argentina, Colombia, España e India.

\section{Oferta}

A partir de la misma encuesta con los laboratorios en el país, también se pudo determinar que la oferta nacional para dar servicios de equivalencia terapéutica de medicamentos es muy limitada. Sin considerar a los fabricantes radicados en Costa Rica que hacen estudios de equivalencia terapéutica de sus propios medicamentos, solo existen dos empresas o laboratorios de ensayos que los brindan, que son LABIOFAR, perteneciente al INIFAR de la Facultad de Farmacia de 
la Universidad de Costa Rica, y CETRATEC- $\alpha$, un laboratorio de capital privado ubicado en el parque industrial de la provincia de Cartago. Asimismo, y debido principalmente al vacío legal ocasionado por la ausencia de una legislación que regule los estudios clínicos en Costa Rica, situación que empezó a subsanarse en abril de 2014 con la aprobación en segunda instancia de la Ley Reguladora de Investigación Biomédica, estos dos laboratorios solo han estado brindando los servicios para realizar perfiles de disolución comparativos (estudios in vitro).

\section{Conclusiones}

En Costa Rica existe una demanda de estudios de equivalencia terapéutica de medicamentos (estudios in vitro e in vivo) que requiere ser satisfecha con prontitud, con el fin de garantizar la calidad, seguridad y eficacia de aquellos que se producen en el país, y, como consecuencia de ello, garantizar su intercambiabilidad, manteniendo el bajo precio y la mayor accesibilidad que caracteriza a los medicamentos genéricos. Asimismo, también se pudo comprobar que la oferta nacional de laboratorios de ensayos que bridan estos servicios, independientes de los fabricantes de medicamentos, es muy limitada.

Por otro lado, en cuanto a la situación particular de los estudios de bioequivalencia (estudios in vivo), los laboratorios fabricantes de medicamentos han tenido que buscar alternativas fuera de Costa Rica para poder satisfacer las exigencias nacionales y del extranjero en cuanto al cumplimiento de requisitos para el registro sanitario de los medicamentos que producen.

Por último, como se pudo apreciar a partir de los datos de la encuesta, se observa una tendencia de los laboratorios fabricantes a realizar ellos mismos los estudios de equivalencia de los medicamentos que fabrican, principalmente en el caso de los estudios in vitro. Con respecto a los estudios in vivo, por el momento no se puede afirmar lo mismo, dado que hasta hace muy poco tiempo la legislación costarricense no permitía realizarlos en el país, si bien dos de estos laboratorios fabricantes dicen tener la capacidad para hacerlos. Este comportamiento, tendencia o aspiración de los laboratorios a realizar sus propios estudios de equivalencia podría explicarse inicialmente por los vacíos que persisten en la legislación nacional, o incluso por el hecho de que en Costa Rica la oferta de estos servicios es muy limitada, ausente y/o eventualmente costosa. Sin embargo, no deja de ser un asunto de particular interés para las autoridades sanitarias y los pacientes usuarios de medicamentos el hecho de que el propio laboratorio fabricante sea juez y parte en la decisión de la intercambiabilidad de los medicamentos que producen.

\section{Agradecimientos}

A la Dra. Rosaura Romero Chacón, Directora del Ciprona de la UCR; a la Dra. Lidiette Fonseca González, Decana de la Facultad de Farmacia de la UCR; a la MBA. Aída Rojas Rojas, profesora UCR; y al Dr. Jorge Pacheco Molina, profesor de la Facultad de Farmacia de la UCR.

Este artículo forma parte del tema de mi tesis -defendida en el 2014-para la obtención del grado de Máster en Química Industrial del Programa de Posgrado en Química de la Universidad de Costa Rica.

\section{Bibliografía}

Abad, F., Novalbos, J. \& Ochoa, M. (2008). Medicamentos genéricos y precios de referencia. En P. Lorenzo, A. Moreno, I. Lizasoain, J. Leza, M. Moro y A. Portolés (Eds.), Farmacología Básica y Clínica de Velázquez (pp. 1305-1313). Madrid: Editorial Médica Panamericana. 
CCSS. (2012). Programa Salud para Todos: Medicamentos. Costa Rica. Obtenido de http://www.youtube.com/ watch? $v=d B y 34 X 0 s-p E$

Dunne, S., Shannon, B., Dunne, C. \& Cullen, W. (2013). A review of the differences and similarities between generic drugs and their originator counterparts, including economics benefits associated with usage of generic medicines, using Ireland as a case study. BMC Pharmacology \& Toxicology, 14(1), 1-19.

EFPIA. (2007). La iniciativa sobre medicamentos innovadores. Bruselas. Obtenido de http://www.medicinesformankind.eu/upload/pdf/IMI_SP.pdf

FDA. (2010). Estudios de biodisponibilidad y equivalencia para productos farmacéuticos administrados oralmente. Consideraciones generales. Obtenido de http://www.fda.gov/Drugs/ GuidanceComplianceRegulatoryInformation/Guidances/ucm201469.htm

FIP. (2012). Biowaiver Monographs 2004-2012: Bringing essential medicines to those who need them most. Obtenido de https://www.fip.org/centennial/files/static/press/FIP_centennialbook_biowaiver_webversion.pdf

IMS Institute. (2011). The Global Use of Medicine: Outlook Through 2015. New Jersey. Obtenido de https:// www.imshealth.com/ims/Global/Content/Insights/IMS\%20Institute\%20for\%20Healthcare\%2OInformatics/ Documents/The_Global_Use_of_Medicines_Report.pdf

Moreno, G., Martín, M., López, A., López, E., Montes, E., González, C., Plasencia, M., Olmo, V. \& Fernández, B. (2010). Genéricos y Bioequivalencia ¿Seguimos dudando? Infarma Servicio Canario de la Salud, 2(2), 1-2.

Moreno, M. (2011). Medicamentos genéricos. Requisitos para su comercialización. En G. Hernández, A. Moreno, F. Zaragozá y A, Porras (Eds.), Tratado de Medicina Farmacéutica (pp. 539-542). Madrid: Editorial Médica Panamericana.

Niazi, S. (2007). Handbook of Bioequivalence Testing. New York: Informa Healthcare.

OPS. (2011a). Guía para la implementación de estrategias de medicamentos genéricos en los países de América Latina y El Caribe como mecanismo para mejorar el acceso a medicamentos. Washington D.C.: OPS. (Serie Técnica de Medicamentos Esenciales, Políticas Farmacéuticas, Documento № 3). Obtenido de http://apps. who.int/medicinedocs/documents/s19196es/s19196es.pdf

OPS. (2011b). Marco para la ejecución de los requisitos de equivalencia para los productos farmacéuticos. Washington D.C.: OPS (Serie RED PARF GT BE Documento Técnico № 8). Obtenido de http://www.paho.org/ hq/index.php?option=com_docman\&task=doc_view\&gid=18848\&ltemid=

Saavedra, I., Iturriaga, V., Ávila, L. \& Quiñones, L. (2011). Estudios de bioexención (in vitro) para establecer equivalencia de medicamentos. Cuadernos Médicos Sociales, 51(2), 66-79.

Vacca, C., Fitzgerald, J. \& Bermúdez, J. (2006). Definición de medicamento genérico ¿un fin o un medio? Análisis de la regulación en 14 países de la región de las Américas. Revista Panamericana de Salud Pública, 20(5), 314-323.

WHO. (2006). Annex 8: Proposal to waive in vivo bioequivalence requirements for WHO Model List of Essential Medicines immediate-release, solid oral dosage forms. OPS (Techical Report Series № 8). Obtenido de http:/l apps.who.int/prequal/info_general/documents/TRS937/WHO_TRS_937_annex8_eng.pdf 